THE ASTROPHYSICAL JOURNAL, 536:308-318, 2000 June 10

(C) 2000. The American Astronomical Society. All rights reserved. Printed in U.S.A

\title{
LIMITS ON THE SPATIAL EXTENT OF ACTIVE GALACTIC NUCLEI MEASURED WITH THE FINE GUIDANCE SENSORS OF THE HUBBLE SPACE TELESCOPE ${ }^{1}$
}

\author{
RICHARD N. HOOK \\ Space Telescope European Coordinating Facility, European Southern Observatory, Karl-Schwarzschild-Strasse 2, D-85748 Garching, Germany; \\ rhook@eso.org \\ ETHAN J. SCHREIER \\ Space Telescope Science Institute, 3700 San Martin Drive, Baltimore, MD 21218; schreier@stsci.edu
}

AND

GeORGe MiLeY

Sterrewacht Leiden, Leiden University, P.O. Box 9513, NielsBohrweg 2, 2300 RA Leiden, Netherlands; miley@strw.leidenuniv.nl Received 1999 October 22; accepted 2000 January 26

\begin{abstract}
The optical structures of several active galactic nuclei (AGNs) have been studied using the fine guidance sensors (FGSs) on the Hubble Space Telescope (HST). The FGSs are interferometric devices that can resolve structure on scales of 20 mas or less and hence have the potential to improve on the resolution attainable by $H S T$ 's cameras. The FGSs produce interferometric fringes known as $\mathrm{S}$ curves, which are related to the intensity profile of the object on the sky. These have been analyzed using a simple model for the radial intensity distribution and strength of the underlying background illumination of the observed objects. Eight different observations of six different AGNs have been analyzed. No statistically significant differences from point sources are detected, but significant upper limits of order 20 mas are placed on any spatial extent. Systematic effects limiting the resolution are discussed, and some simple conclusions about the physical size and luminosity densities of the emitting regions of the AGNs implied by the data are given.

Subject headings: galaxies: active — galaxies: nuclei — methods: data analysis techniques: interferometric
\end{abstract}

\section{INTRODUCTION}

One of the most important properties of the Hubble Space Telescope (HST) is its ability to attain high spatial resolution limited by diffraction rather than the properties of the atmosphere. This resolution is normally exploited via direct imaging by $H S T$ 's cameras, potentially enhanced by the application of restoration algorithms. The fine guidance sensors (FGSs) of the HST offer an alternative method for studying structure on the finest scales. The FGSs fold onehalf of the telescope's pupil onto the other, and the resulting interference fringes are recorded by photomultiplier tubes as the 5" FGS field of view is scanned across a source. These fringes are the basis of the HST's high-precision tracking but can also be used to study structure on scales down to 20 mas or less. The FGSs are more sensitive than the cameras to the finest spatial structure but produce data that are more difficult to interpret.

We have carried out a project to investigate the usefulness of the HST FGSs for attaining ultrahigh resolutions on extragalactic objects, observing several bright AGNs known to have radio structure on scales ranging from several milliarcseconds to several tens of milliarcseconds. The goal was to search for and study similar scale structure in the optical.

In this paper we present the results of these observations using the original HST FGSs and their analyses using a simple model for detecting and quantifying extended struc-

\footnotetext{
${ }^{1}$ Based on observations made with the NASA/ESA Hubble Space Telescope, obtained from the Space Telescope Science Institute, which is operated by the Association of Universities for Research in Astronomy, Inc., under NASA contract NAS5-26555.
}

ture. We apply statistical tests to decide whether the hypothesis that the object is a point source on an extended background is consistent with the data and if so, at what confidence level. Simulations are used to derive limits on the detection thresholds for extensions to the central point source. The systematic effects due to calibrations incorrectly matched in both time and color are investigated, as are other systematic effects that limit the resolution we can attain. We find that all our data sets are consistent with pointlike object intensity distributions. The high spatial resolution of the FGS allows us to derive interesting limits on the physical sizes of the optical emission regions and on the scale and relative intensities of features that would have been seen. These results delineate the region of phase space where the FGSs are the best observational tool for mapping at high resolution in the optical, especially given the improved characteristics of the newly available FGSs.

\section{DATA FROM THE FGS}

The HST uses three FGSs at the edge of the field of view of the telescope (see Nelan \& Makidon 1999 for further discussion). During normal use of the telescope, two of the FGSs are used to "lock" onto guide stars to precisely point the telescope, while the third is available to perform astrometry, independently scanning objects within its field of view and measuring their positions. Each FGS contains a pair of orthogonal interferometers, which, when scanned across a point source, produce two S-shaped "transfer functions." The zero-point crossings of the transfer functions determine the position of the source - these nulls are used by the pointing control of the telescope-while the morphology of the transfer functions is dependent on the brightness profile 


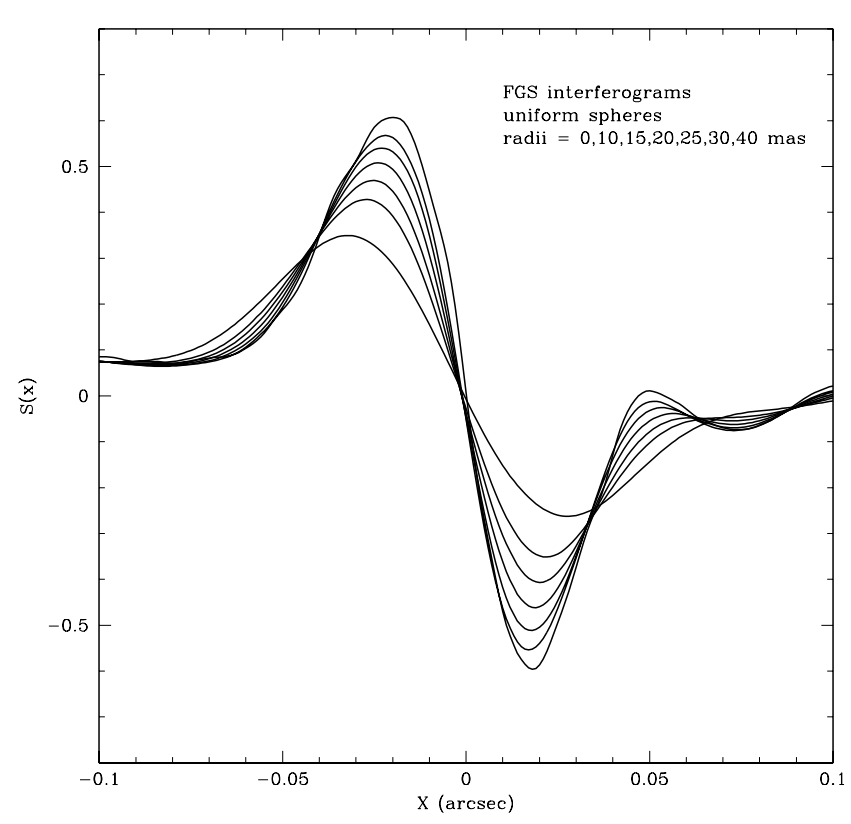

FIG. 1.-Predicted HST FGS S curves for uniform, optically thin spherical sources of the radii shown. A point source produces an $S$ curve with the highest amplitude. More extended objects result in $\mathrm{S}$ curves of smaller amplitude and greater width.

of the source. Extended or multiple objects produce lower amplitude and more complicated transfer functions compared with those from a point source. It may be shown that the $S$ curve is related to the monochromatic intensity distribution of the object on the sky in the following way:

$$
S(\theta)=\frac{I_{A}-I_{B}}{I_{A}+I_{B}}=\int_{-\Delta \phi / 2}^{\Delta \phi / 2} \frac{F(\phi)}{F_{\text {tot }}} \frac{\sin ^{2}[D \pi(\theta-\phi) / \lambda]}{D \pi(\theta-\phi) / \lambda} d \phi,
$$

where $S(\theta)$ is the value of the observed $S$ curve expressed as a function of angle away from interferometric null, $I_{A}$ and $I_{B}$ are the intensities measured by the photomultipliers, $D$ is the aperture of the HST, $\lambda$ is the wavelength, $F(\phi)$ is the intensity distribution of the object in the direction of the scan, and $F_{\text {tot }}$ is the total integrated flux. The observed $S$ curve can be regarded as the convolution of the normalized intensity profile of the object on the sky and the $S$ curve resulting from a reference observation of a point source on a negligible background. It follows from equation (1) that, in principle, the morphology of the source (in the direction of the scan) can be obtained by deconvolving the transfer function from the observed FGS interferometer output. A method for doing this has been described by Hershey (1992). Stronger backgrounds lead to lower amplitude $S$ curves, and this effect becomes important for faint sources such as those discussed in detail later in this paper. To illustrate the general form of the FGS $S$ curves and how they change when the object has small extent, simulations were created and the results are shown in Figure 1.

The primary use of the FGS in transfer function mode is for observations of binary stars (see, e.g., Franz et al. 1992), where separations, position angles, and relative intensities of close binaries are measured. Lattanzi et al. (1997) have also successfully used the FGS to measure the diameters of the disks of Mira variables. The observations discussed in this paper are the first to attempt to study extended structures in extragalactic sources with the FGS.

\section{THE OBSERVATIONS AND DATA REDUCTION}

A list of data sets used in the analysis described below is given in Table 1. All observations were made with FGS3 in the TRANS mode (see Nelan \& Makidon 1999) to obtain transfer functions. The initial observations in this survey were carried out with three different filters: PUPIL (a filterfree stopped-down aperture), CLEAR (a broadband filter with FWHM of $2340 \AA$ centered at $5830 \AA$ ), and YELLOW (a $750 \AA$ FWHM filter centered at $5500 \AA$ ). The original goal was to attempt to derive some color information from the filters, while the pupil stop, which apodizes the outer one-third of the telescope pupil, was used to restore fringe visibility in the presence of spherical aberration. Although in principle, the interferometers should not be affected by the symmetric aberration in the primary mirror, small internal FGS misalignments interact with the spherical aberration to impair FGS performance. All later observations and all the data analyzed here used the pupil stop only.

Each observation consisted of multiple scans across the object. The two orthogonal interferometers of the FGS were oriented at $45^{\circ}$ to the scan direction. Each scan was typically 2 ".4 long, centered on the object, with a nominal 0.3 mas step size.

Routine data reduction software developed by the FGS group at STScI, working with the HST Astrometry team, was used to unpack, inspect, smooth, and merge the data sets (see, e.g., Baum 1994). Individual scans that, on visual inspection showed no obvious transfer function or displayed pathological (nonphysical) characteristics due to spacecraft jitter, were not considered further. On the average, $75 \%$ of the scans were considered "good." The data from these scans were then co-added, separately for each filter and for each coordinate axis. We used the standard merging software, which first determines a "zero phase" for each scan by measuring the zero crossing of the transfer function (observed in the smoothed data), then calculates the relative offsets of the observed transfer functions from that of a reference scan (typically the first scan of the

TABLE 1

OBSERVED FGS AGN DATA SETS

\begin{tabular}{|c|c|c|c|c|c|}
\hline Object Name & Data Set Name & Observation Date & Exposure Time (s) & Signal Strength & Calibration Data Set \\
\hline 3C 279 . & f0wj0602m & 1992 Apr 2 & 1575 & strong & f0v30302m \\
\hline NGC $1275 \ldots . .$. & f0wj0203m & 1992 Oct 27 & 1508 & very weak & f0v30702m \\
\hline $3 C 345 \ldots \ldots \ldots \ldots$ & f0wj0702m & 1993 Apr 12 & 1590 & weak & $\mathrm{f} 0 \mathrm{v} 30 \mathrm{a} 02 \mathrm{~m}$ \\
\hline NGC $4151 \ldots \ldots$. & $\mathrm{f} 2 \mathrm{~m} 40201 \mathrm{~m}$ & 1995 Jan 29 & 2596 & very strong & $\mathrm{f} 2 \mathrm{vc} 0201 \mathrm{~m}$ \\
\hline $3 \mathrm{C} 345 \ldots \ldots \ldots \ldots$ & $\mathrm{f} 2 \mathrm{~m} 40501 \mathrm{~m}$ & $1995 \mathrm{Feb} 22$ & 1811 & weak & $\mathrm{f} 2 \mathrm{vc} 0201 \mathrm{~m}$ \\
\hline $3 \mathrm{C} 273 \ldots \ldots \ldots$ & $\mathrm{f} 2 \mathrm{~m} 40302 \mathrm{~m}$ & 1995 Mar 21 & 1096 & very strong & $\mathrm{f} 2 \mathrm{vc} 0201 \mathrm{~m}$ \\
\hline $3 \mathrm{C} 279 \ldots \ldots \ldots$ & $\mathrm{f} 2 \mathrm{~m} 40401 \mathrm{~m}$ & 1995 Apr 10 & 1811 & strong & $\mathrm{f} 2 \mathrm{vc} 0201 \mathrm{~m}$ \\
\hline 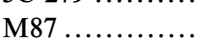 & $\mathrm{f} 2 \mathrm{~m} 40101 \mathrm{~m}$ & 1995 May 12 & 2596 & very weak & $\mathrm{f} 2 \mathrm{vc} 0201 \mathrm{~m}$ \\
\hline
\end{tabular}


TABLE 2

List of Calibration Data Sets of Reference Stellar Sources

\begin{tabular}{cllc}
\hline \hline Star & Data Set & Observation Date & Color $(B-V)$ \\
\hline Upgren 69....... & f0v30302m & 1992 Apr 6 & +0.5 \\
Upgren 69....... & f0v30702m & 1992 Sep 1 & +0.5 \\
Upgren 69........ & f0v30a02m & 1993 Apr 5 & +0.5 \\
Upgren 69........ & f2vc0201m & 1995 Sep 12 & +0.5 \\
Upgren 69........ & f2cs0502m & 1994 Jun 12 & +0.5 \\
Upgren 69....... & f3h80201m & 1996 Oct 23 & +0.5 \\
Upgren 69....... & f3h80501m & 1997 Jan 24 & +0.5 \\
Upgren 69....... & f3h85501m & 1997 Mar 30 & +0.5 \\
LAT-COL-1B...... & f2vu0401m & 1996 Jan 9 & +0.18 \\
\hline
\end{tabular}

set), and then co-adds the raw (unsmoothed) data from the individual scans, using the calculated offsets. For the data from the faint objects discussed here each individual scan has significant photon noise. This limits the accuracy of the offset determination and degrades the resolution that can finally be attained. This source of systematic error is discussed below in $\S 5$.

It was apparent that the pupil data were the "cleanest" in the sense that the amplitudes of the transfer functions were greatest and the shapes were smoothest and most closely resembled expected transfer function shapes. This is expected because the full aperture, which accepts a larger portion of the spherically aberrated optical beam, is more susceptible to internal FGS misalignments. In order to have a uniform set of data sets for many objects, which could be adequately calibrated with the limited set of calibration curves at our disposal, we decided to restrict the analyses presented here to those data sets obtained using the pupil stop. A total of six observations of the objects studied here did not use the pupil stop and were not suitable for further analysis. Unfortunately, use of the pupil did reduce the photon flux and thus the number of sources we could study with this technique, as well as the angular resolution that could be achieved. Future observations with refurbished FGSs will not require the pupil stop and will eliminate these problems.

In order to analyze these curves, we require for reference the response of the system to a known point source on a negligible background, effectively the S-curve point-spread function (PSF). The FGS is routinely scheduled to observe standard stars of different colors, through different filters, to provide such references. We selected as reference $S$ curves for our analyses observations of stars observed through the pupil aperture as close as possible in time to our observations, as the FGS3 S curves are known to change over the course of time. Ideally a reference star with color comparable to that of the object should be selected, but for AGNs this can only be approximated. It is also not always possible to find reference curves taken less than about 200 days from a given observation. The systematic effects due to the inadequacies of the reference curves in color and time are discussed below in $\S 5$. A list of all calibration curves used in this paper is given as Table 2.

\section{MODEL FITTING THE OBSERVED S CURVES}

The merged transfer functions for several objects observed in our sample appeared clearly inconsistent with what would be expected from an ideal point source. In particular, the amplitudes of the $S$ curves were less (suggesting that the background was a significant fraction of the object intensity) and the widths appeared greater (suggesting extent) than expected for point sources. To understand the significance of these differences, we created model object intensity profiles, generated sets of simulated $S$ curves from them, and compared these to the observations. In this section we use the 3 C 279 data set f0wj0602m to illustrate the methods used to assess the statistical significance of the structures seen in the $S$ curves. The other data sets were analyzed in the same way, and the results are presented below.

The objects are modeled using normalized Gaussian intrinsic intensity profiles, superimposed on flat backgrounds. The two-parameter set of functions we used for the intensity distribution in each of $x$ and $y$ is thus of the form

$$
F=B+H e^{-\theta^{2} / 2 \sigma^{2}},
$$

where $B$ and $H$ are constrained so that the sum over all values of $F$ is $1.0, \theta$ is the angle with respect to the FGS interferometry null (normally expressed in milliarcseconds), and $\sigma$ is expressed in the same units. The normalization of this function is important and may be derived easily from the theory of the FGSs (see eq. [1]). This function $F$ is a model for the intensity profile of the object in one direction, summed over the $5^{\prime \prime}$ width of the FGS instantaneous field of view in the perpendicular direction. The model $S$ curve itself is then created by convolving the model source profile $F$ with the appropriate calibration $S$ curve, the PSF, as listed in Table 1.

The observation data sets were reduced using the standard FGS software referenced earlier. The noisy, unsmoothed data products rather than the smoothed versions were used (both are created by the standard FGS software), as we wished to retain the noise properties of the data. For the calibrator $S$ curves, the smoothed version was used, resampled to match the scale and range of the observed data. In both cases a 512 sample subset of the data around the central S-curve feature was used to make the convolutions more efficient. This reduced range does not affect the results, as there are no significant features farther out in these data.

The observed data sets were compared with the models (convolutions of the intensity profiles with calibration $\mathrm{S}$ curves) for a range of values of $\sigma$ and $B$, and the sum of squared residuals was computed. To estimate the statistical significance of the results, we measure the noise in the outer parts of the $S$ curve, where the mean is zero, and assume it to be approximately constant. The reduced $\chi^{2}$ for the fits is then calculated in the normal way:

$$
\chi_{\text {red }}^{2}=\frac{\sum\left(v_{\text {dat }}-v_{\text {model }}\right)^{2} / \mathrm{var}}{n_{\text {free }}},
$$

where $v_{\text {dat }}$ and $v_{\text {model }}$ are the values of the data and model at a given position, $n_{\text {free }}$ is the number of degrees of freedom, in this case the number of data points (512) minus the two free fit parameters (the width of the Gaussian and the fraction of energy in the background), and var is the measured variance of the noise, assumed to be constant throughout the $S$ curve. It is assumed that there is no correlation between the data points; this assumption will be discussed further below.

Confidence levels are plotted in Figure 2 for the twoparameter fits to the 3C 279 data sets (f0wj0602m). For a $\chi^{2}$ 

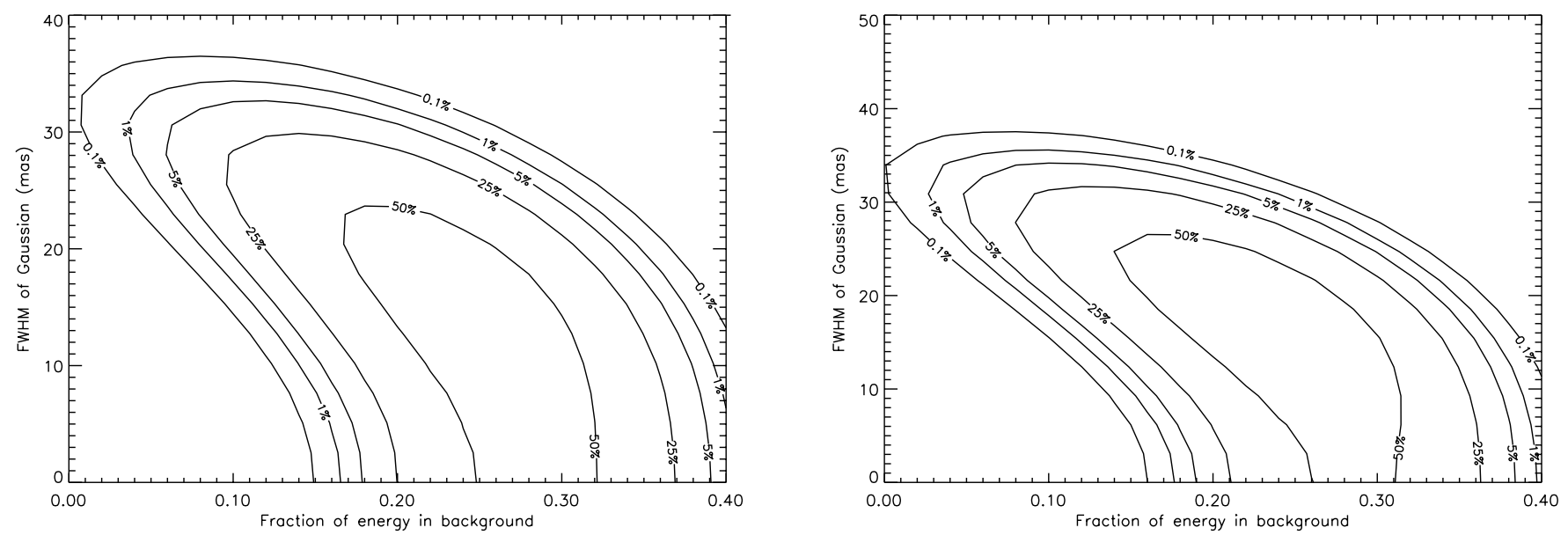

FIG. 2.-Confidence limits of fits of a two-parameter set of models of a Gaussian and a flat background to the $x$ - and $y$-direction S curves for 3C 279 data set f0wj0602m.

distribution with 510 degrees of freedom a reduced $\chi^{2}$ of 1.072 corresponds to the $25 \%$ confidence level, 1.123 to $5 \%$, and 1.161 to $1 \%$. The reduced $\chi^{2}$ values of the good fits are slightly less than 1.0, implying that the noise as estimated from the outer parts of the $S$ curve is slightly too large, in agreement with visual inspection; the data are not "overfitted," as the curve is smooth on large scales and cannot fit any but the largest features in the $S$ curve. Estimating the variance from the outer parts of the $S$ curve gives a spread around the truth with the effect of shifting the confidence contours in or out. We use the $F$-test (below), based on ratios of $\chi^{2}$ values, as it is not affected by small variations of the variance measure.

For statistical tests to be valid, we need to know the magnitude of the noise, its distribution, and whether there are correlations present that would reduce the effective number of degrees of freedom. To assess these properties, we analyzed a "noise data set" produced from the difference between the fitted and observed S curves for 3C 279 (the $y$ data were used but $x$ would be equally good). Again, because the fitted curve is smooth on all but the largest scales, this will be a good guide to the fine structure of the noise. The histogram of this noise data set appeared approximately Gaussian with zero mean, a width (i.e., the $\sigma$ of the noise) of 0.044 , and no obvious asymmetry. Figure 3 shows the power spectrum of the noise, which appears to be

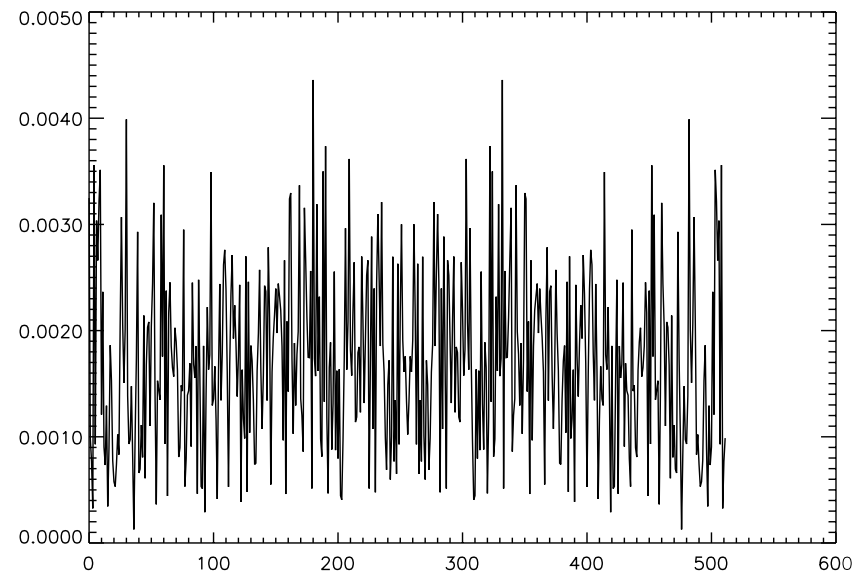

FIG. 3.-Power spectrum of the noise in a typical $\mathrm{S}$ curve approximately "white." It is plotted with the zerofrequencybin at the center and is hence symmetrical about this point. Finally, the correlations within the noise were assessed by block averaging the data in 2,4 , and 8 sample bins and computing the standard deviation in each. If there are no correlations on these scales, the values should drop by $\sqrt{2}$ each time. The actual ratios found were 1.50,1.47, and 1.36, confirming that the noise may be regarded as Gaussian and independent, with 512 degrees of freedom.

As seen from Figure 2, there is a large region of acceptable parameter space. The best fit requires a small extension (FWHM about 15 mas) with a background of about $24 \%$. However, a point source with a background about $28 \%$ of the total intensity is also fully acceptable; the required background is consistent with the level expected from an object of this magnitude. The hypothesis that there is no background and that the reduction in S-curve amplitude is due to a broad nucleus is strongly rejected at a significance level of $99.9 \%$.

We adopt two approaches to further assess the significance of the small extension indicated by the minimum $\chi^{2}$ fit. First, we ask whether the improvement in the fit due to the addition of an extra parameter to the model (the extent of the object) is justified by the data. We compare the $\chi^{2}$ of the null hypothesis "the object is a point" with that obtained by including a nonzero width as well. The ratio of these two $\chi^{2}$ values should follow the $F$-distribution, allowing us to put a confidence limit on the extension. The use of the $F$-test also removes the uncertainty in the measurement of the variance, as we are comparing a ratio of reduced $\chi^{2}$ values.

From the 3C 279 S curves we have

$$
F=\frac{\chi_{\text {extended }}^{2}}{\chi_{\text {point }}^{2}}=0.9807 / 0.9682=1.013
$$

for $x$ and

$$
F=\frac{\chi_{\text {extended }}^{2}}{\chi_{\text {point }}^{2}}=0.9892 / 0.9573=1.033
$$

for $y$, where there are 511 degrees of freedom for the pointsource fit and 510 for the two-parameter fit. For an $F$ distribution with 510 and 511 degrees of freedom, a $10 \%$ confidence level is reached at $F_{510,511,0.9}=1.12$. In other 

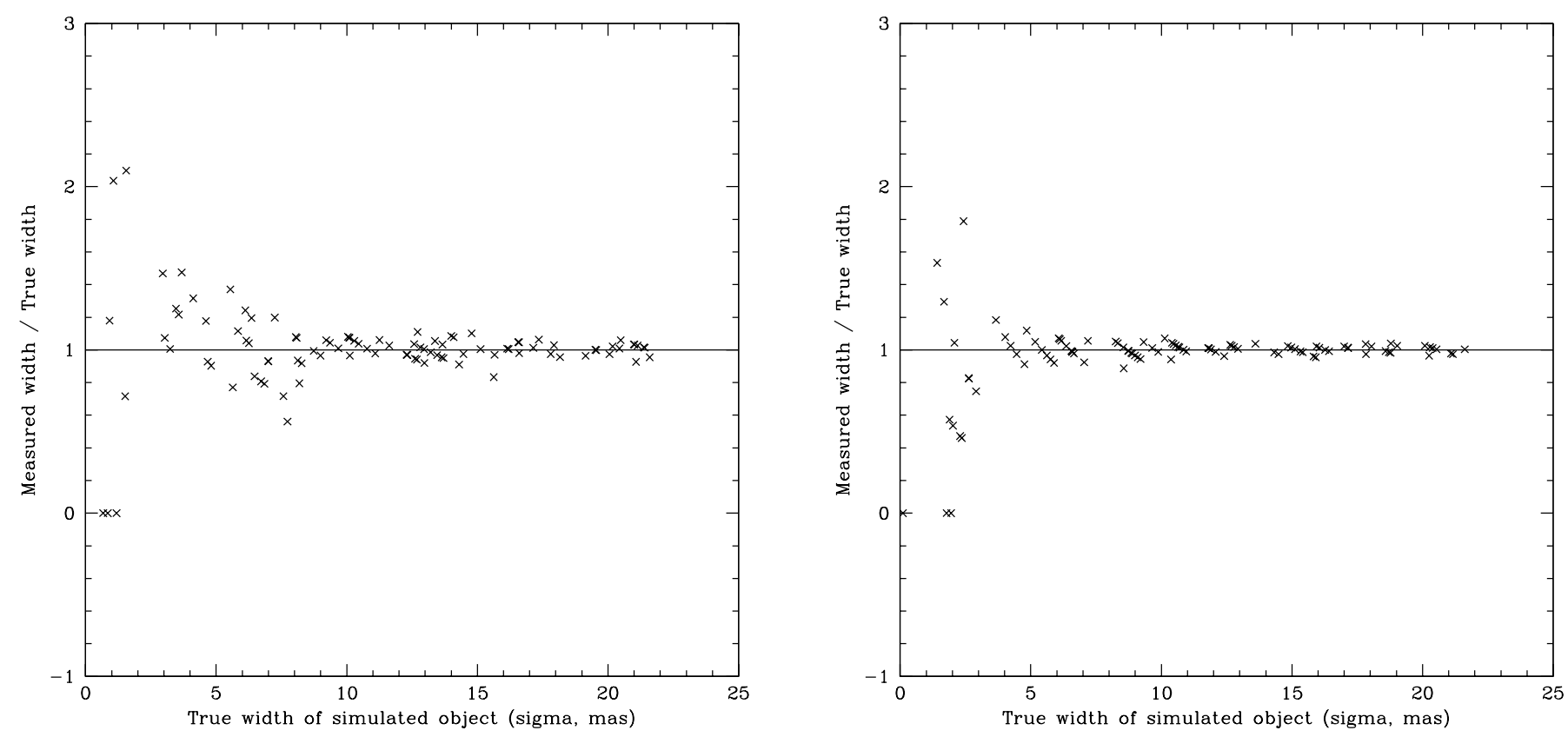

FIG. 4.- Ratio of measured to true object extension as a function of object width for simulations at two different noise levels. The data had noise levels of (left panel) 0.05 and (right panel) 0.01 . The simulations are described further in the text.

words, such a high value is to be expected by chance in $10 \%$ of cases if the null hypothesis were true. Although this application of the $F$-statistic is not rigorously correct, as the fitted function is not linear in the width of the Gaussian, we think it is clear that we cannot reject the null hypothesis and hence cannot put any trust in an extension when the derived $F$-values are so close to 1.0 .

In a second approach, we generate Monte Carlo simulations using the same two-parameter model, selecting the width and fractional background strength at random, convolving the models with the same calibrator $S$ curve, and adding Gaussian noise at levels comparable to that present

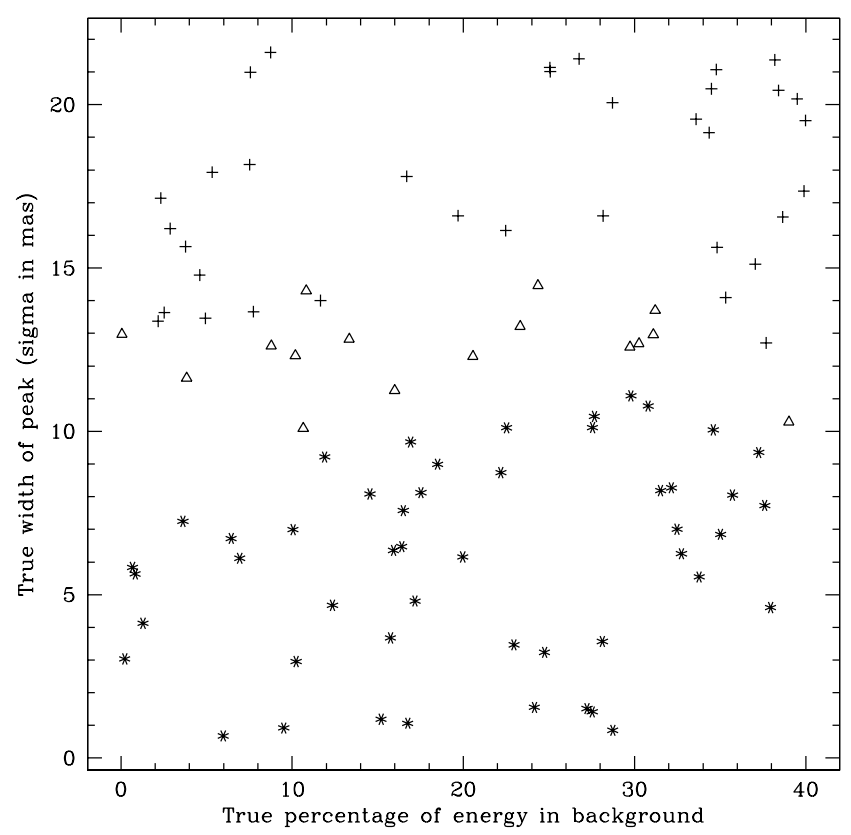

in two data sets of interest. The results were analyzed in exactly the same way as above to measure the width and background. Figure 4 compares the measured widths with those input to the simulations for two noise levels. For the noisier simulations on the left, which have noise comparable to that seen in the $3 \mathrm{C} 279 \mathrm{~S}$ curves, there is reasonable agreement for extents $(\sigma)$ greater than about 10 mas. In a higher signal-to-noise ratio $(\mathrm{S} / \mathrm{N})$ case on the right, where the noise is comparable to that seen in the NGC 4151 data set, extents down to about 5 mas can be reliably detected. These plots provide a guide to whether or not an extension can be detected at a specified noise level when other sources

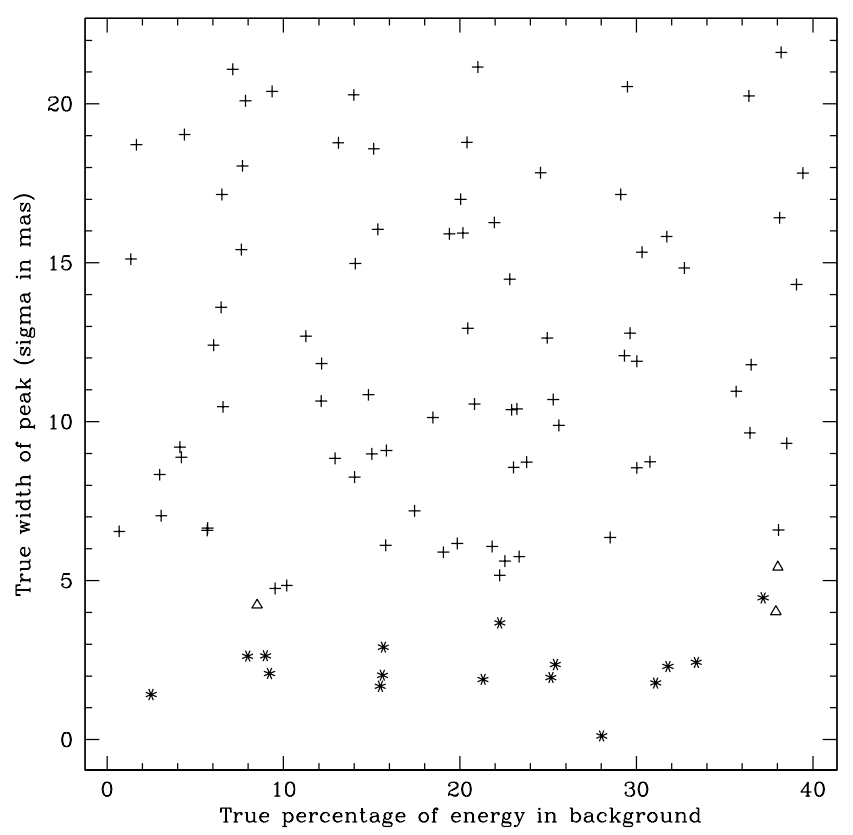

FIG. 5.- Simulated $S$ curves measured and classified by whether they are significantly nonpointlike or not. The plus signs represent fits that are nonpointlike at the $99 \%$ confidence level. Noise levels were (left panel) 0.05 and (right panel) 0.01 . See the text for more details. 
of systematic error are negligible. We quantify the statistical significance of the extent determination by again looking at the $F$-statistic for each of these $S$ curves, as plotted in Figure 5 . The plus signs indicate fits that are nonpointlike at the $99 \%$ confidence level and the stars fits that have $F$-values insufficiently different from 1.0 to allow rejection of the null hypothesis (i.e., point source) at the $25 \%$ level. The triangles are intermediate cases.

It is clear from the left-hand panels that $\sigma$ values less than about 12 mas (FWHM = 28 mas) will not be detectable at statistically significant levels in S curves having noise levels comparable to the $3 \mathrm{C} 279$ data. Hence, the extension of $\sigma=6$ mas (FWMH $=14$ mas) we obtained for the 3C $279 x$ $S$ curve is not significant. Similarly the smaller extension measured for the $y \mathrm{~S}$ curve of the NGC 4151 data described later in the paper is also not statistically significant. In this case, that of the best $\mathrm{S} / \mathrm{N}$ of our sample, the smallest statistically significant extent $(\sigma)$ detectable is of order 5 mas. For data of lower $\mathrm{S} / \mathrm{N}$, including most of the other data sets studied here, the minimum extension that can be detected will clearly be larger than that for 3C 279 . Figures 4 and 5 also show that the minimum detectable extension falls as the fraction of energy in the background increases. This is to be expected, as the amplitude of the $S$ curve, compared with the constant noise, is also falling in this case.

These simulations allow us to place limits on the spatial extents of the objects in our study that would be detected at different noise levels under the assumption that other systematic effects are negligible. Unfortunately the combined effects of the spherical aberration of the HST, small misalignments within FGS3 itself, variations in the fringe shapes with time, and the impossibility of accurately coadding shifted fringes with very low signal restrict the resolution that can be obtained. These limits are discussed below.

\section{SYSTEMATIC EFFECTS}

The above analysis has assumed that the calibration reference $S$ curves are an accurate representation of what would be seen if the observed object were a point on a negligible background. It is also assumed that the observed $S$ curves can be regarded as the convolution of the normalized object intensity distribution with the calibration $S$ curve. Unfortunately neither of these assumptions can be regarded as completely valid for $S$ curves of faint objects observed with the FGS3 interferometer. In practice, S curves depend on the color of the object and are also known to vary in time. In addition, the accuracy of aligning and co-adding the multiple scans across a faint object is limited by the photon noise of each scan. In this section we discuss how these systematic effects limit our results.

The effect of color on the $S$ curve follows simply from the definition of the transfer function: equation (1) contains the dependence of the $S$ curve on color. For a monochromatic $S$ curve, changing the wavelength has the effect of scaling the $S$ curve with respect to the $\theta$ axis. This is a simple effect that can be modeled for an object of known spectral-energy distribution by also convolving over wavelength. We generated two simulated $S$ curves based on two extremes, a very blue spectrum and a very red spectrum. We then used the red $S$ curve as a calibrator to analyze the blue one using the procedures discussed above. The color difference, far larger than that in any realistic case, is found to introduce a ripple in the residuals of the best fit but no measurable extent.

We also took the spectrum of NGC 4151 and multiplied by a simulated FGS spectral response and used this to generate a simulated $S$ curve corresponding to a point source having the appropriate spectral energy distribution (SED). We then made another simulated $S$ curve with spectral weighting appropriate for the calibration star Upgren 69. To test the color effect on our results we analyzed this simulated NGC 4151 observation using the simulated Upgren $69 \mathrm{~S}$ curve as calibrator. The resultant fit showed low-amplitude residuals but no detectable bias in the measurement of extent or background, both of which were zero at the resolution of the analysis.

The time variation of the $S$ curves is much less well understood than the color dependence. It presumably depends on time-dependent changes in the geometry of the fine guidance sensors. Experience with FGS3 data on double stars and the disks of Mira stars has shown that the variations, which are particularly marked for scans in $x$, limit resolution to approximately 20 mas. Unfortunately, there are at present insufficient data to allow predictions of this effect. This and other sources of systematic effects are discussed in Nelan \& Makidon 1999.

To empirically estimate the effects of time-variant systematics on the analyses of our data sets as described above, we reprocessed a high $\mathrm{S} / \mathrm{N}$ case (f2m40301, 3C 273) using a set of different reference calibration $S$ curves listed in Table 2, which were taken after the first HST servicing mission. These cover a period from 280 days before the observation to 740 days afterward. The last calibration set listed, below the line, is a bluer star. The resultant fits are given in Table 3.

There is significant variation in quality of fit when different calibration curves are used. As expected, the reference curve closest in time to the observation (f2 vc0201m) does

TABLE 3

Fits to 3C 273 S Curves Using Different Calibration Curves

\begin{tabular}{|c|c|c|c|c|c|c|}
\hline \multirow[b]{2}{*}{ Calibration Data Set } & \multicolumn{3}{|c|}{$x$} & \multicolumn{3}{|c|}{$y$} \\
\hline & $\begin{array}{c}\text { Background } \\
(\%)\end{array}$ & $\begin{array}{c}\sigma \\
(\mathrm{mas})\end{array}$ & $\chi_{\text {red }}^{2}$ & $\begin{array}{c}\text { Background } \\
(\%)\end{array}$ & $\begin{array}{c}\sigma \\
(\mathrm{mas})\end{array}$ & $\chi_{\mathrm{red}}^{2}$ \\
\hline $\mathrm{f} 2 \mathrm{cs} 0502 \mathrm{~m} \ldots \ldots \ldots \ldots$ & 0 & 0 & 2.50 & 0 & 0 & 2.29 \\
\hline $\mathrm{f} 2 \mathrm{vc} 0201 \mathrm{~m} \ldots \ldots \ldots \ldots$ & 0 & 1 & 1.67 & 0 & 3 & 1.47 \\
\hline $\mathrm{f} 3 \mathrm{~h} 80201 \mathrm{~m} \ldots \ldots \ldots \ldots$ & 0 & 0 & 1.26 & 0 & 3 & 1.62 \\
\hline $\mathrm{f} 3 \mathrm{~h} 80501 \mathrm{~m} \ldots \ldots \ldots$ & 0 & 0 & 2.19 & 2 & 1 & 1.45 \\
\hline $\mathrm{f} 3 \mathrm{~h} 85501 \mathrm{~m} \ldots \ldots \ldots$ & 2 & 0 & 1.86 & 0 & 4 & 1.66 \\
\hline f2vu0401m .......... & 0 & 0 & 1.43 & 0 & 4 & 1.78 \\
\hline
\end{tabular}


seem to give a better fit than those obtained a long period either before or after, but the differences are small. All fits give very similar numerical values for both the fitted parameters, on both axes, within the formal uncertainties as calculated before. In this case we find consistently that both the background level and the width are negligible.

A final important source of systematic error is introduced by the reduction of the multiple FGS scans. Each of these has a very low $\mathrm{S} / \mathrm{N}$ for the faint objects considered here, and each has a random, unpredictable shift relative to the others. To co-add these scans, as described in $\S 3$ above, it is necessary to measure their relative shifts. This process cannot be done accurately when noise is very significant, and this process inevitably degrades the resolution attainable.

The systematic effects described above combine to limit the resolution, and hence the minimum detectable object extent, to approximately 20 mas and are greater than the more fundamental limits because of the pure photon noise in the observed S curves. This limit applies to the FGS3 interferometer when used in pupil mode. The newer FGS1R interferometer, installed during the second $H S T$ servicing mission, has been shown to have much more stable $S$ curves and also does not require the use of the pupil stop. Hence, it should have much improved performance for this kind of work.

\section{RESULTS FOR INDIVIDUAL OBJECTS}

All the objects listed in Table 1 were fitted to the twoparameter family of models described above, and the minimum $\chi^{2}$ fit results are shown in Table 4. The data are shown in Figures 6-13. In all figures, the best fit is shown along with the data in the upper panel and the residuals are plotted below, on the same scale. Only the 512 points used in the analysis are plotted. In each subsection below, we summarize the results for each object.

\section{1. $3 C 279$ (Figures 6 and 7)}

$3 \mathrm{C} 279$ is an extended double radio source that includes a compact core and a jet that extends about $5^{\prime \prime}$ (de Pater \& Perley 1983) as well as structure observed with VLBI on scales down to 0.1 mas (Bååth et al. 1992). The jet of 3C 279 was one of the first known examples of superluminal motion. 3C 279 is associated with a quasar at redshift 0.538 (Sandage \& Wyndham 1965). It is a luminous X-ray and gamma-ray source and is highly variable at all wavelengths. In the optical it is highly polarized, with $V$ magnitude ranging from 15 to $17 \mathrm{mag}$ during recent years.

There are two observations of this object, and the earlier ones were used in the above examples of modeling methods. The data are of reasonable quality, and the fits are good. The data are consistent with a point source on a moderate background, consistent with the object's brightness.

\subsection{NGC 1275 (Figure 8)}

These data are of very low signal-to-noise ratio, and the amplitude is very low because of the relatively high background level from this bright galaxy. The fits are reasonable and consistent with a point source.

TABLE 4

Results of Two-Parameter Fits to S Curves

\begin{tabular}{|c|c|c|c|c|c|c|c|}
\hline \multirow[b]{2}{*}{ Data Set Name } & \multirow[b]{2}{*}{ Object Name } & \multicolumn{3}{|c|}{$x$} & \multicolumn{3}{|c|}{$y$} \\
\hline & & $\begin{array}{c}\text { Background } \\
(\%)\end{array}$ & $\begin{array}{c}\sigma \\
(\mathrm{mas})\end{array}$ & $\chi_{\text {red }}^{2}$ & $\begin{array}{c}\text { Background } \\
(\%)\end{array}$ & $\begin{array}{c}\sigma \\
\text { (mas) }\end{array}$ & $\chi_{\mathrm{red}}^{2}$ \\
\hline f0wj0602m ...... & $3 C 279$ & 24 & 5.4 & 1.10 & 28 & 7.2 & 1.03 \\
\hline f0wj0203m ...... & NGC1275 & 80 & 5.5 & 0.83 & 86 & 0.0 & 0.80 \\
\hline f0wj0702m ...... & $3 \mathrm{C} 345$ & 56 & 5.4 & 0.91 & 58 & 7.6 & 1.18 \\
\hline $\mathrm{f} 2 \mathrm{~m} 40201 \mathrm{~m} . . . .$. & NGC 4151 & 22 & 0.0 & 1.74 & 24 & 3.1 & 2.6 \\
\hline $\mathrm{f} 2 \mathrm{~m} 40501 \mathrm{~m} \ldots \ldots$ & $3 \mathrm{C} 345$ & 72 & 0.0 & 0.99 & 68 & 3.2 & 0.91 \\
\hline $\mathrm{f} 2 \mathrm{~m} 40302 \mathrm{~m} \ldots \ldots$ & $3 \mathrm{C} 273$ & 0 & 0.0 & 1.43 & 0 & 2.1 & 1.50 \\
\hline $\mathrm{f} 2 \mathrm{~m} 40401 \mathrm{~m} \ldots \ldots$ & $3 \mathrm{C} 279$ & 18 & 7.6 & 1.23 & 22 & 7.3 & 0.91 \\
\hline $\mathrm{f} 2 \mathrm{~m} 40101 \mathrm{~m} \ldots \ldots$ & M87 & 92 & 13. & 1.20 & 92 & 8.5 & 1.03 \\
\hline
\end{tabular}
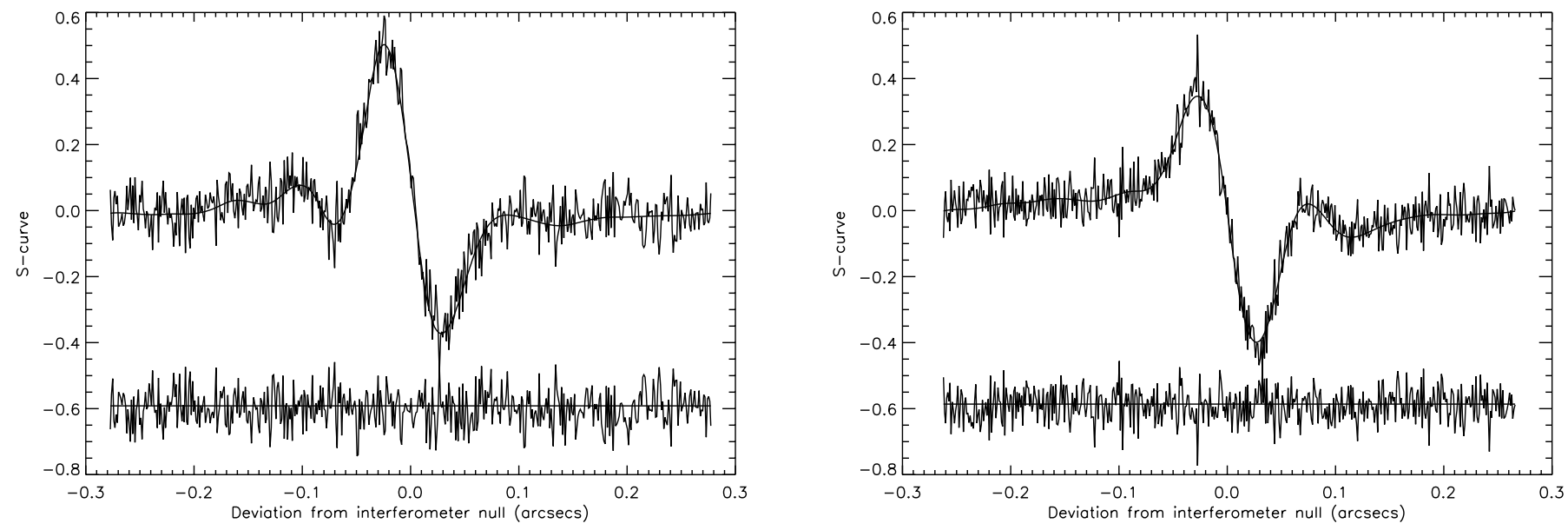

FIG. 6.-Best fit to 3C 2791992 April $2 x$ and $y$ S curves 

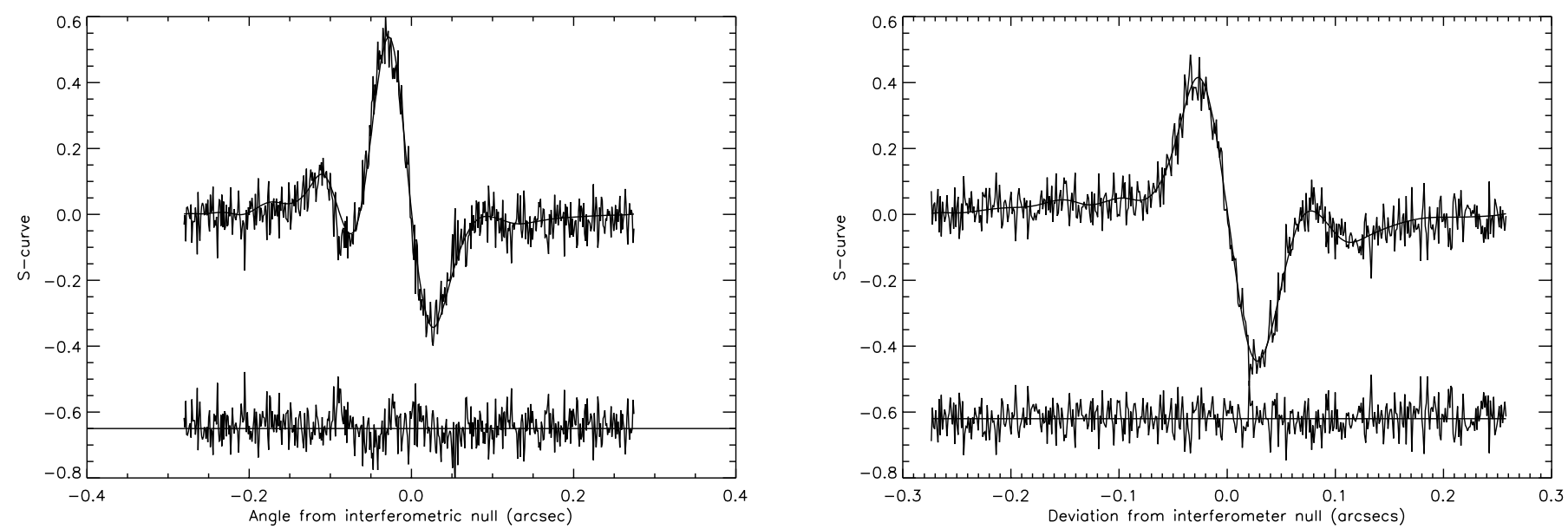

Fig. 7.-Best fit to 3C279 1995 April $10 x$ and $y$ S curves

6.3. $3 C 345$ (Figures 9 and 10)

Both data sets for this object have low $\mathrm{S} / \mathrm{N}$. The fits are good and are consistent with point sources.

6.4. NGC 4151 (Figure 11)

These $S$ curves have excellent $\mathrm{S} / \mathrm{Ns}$, the best of all those studied here. Unfortunately the fits are not very good, as is

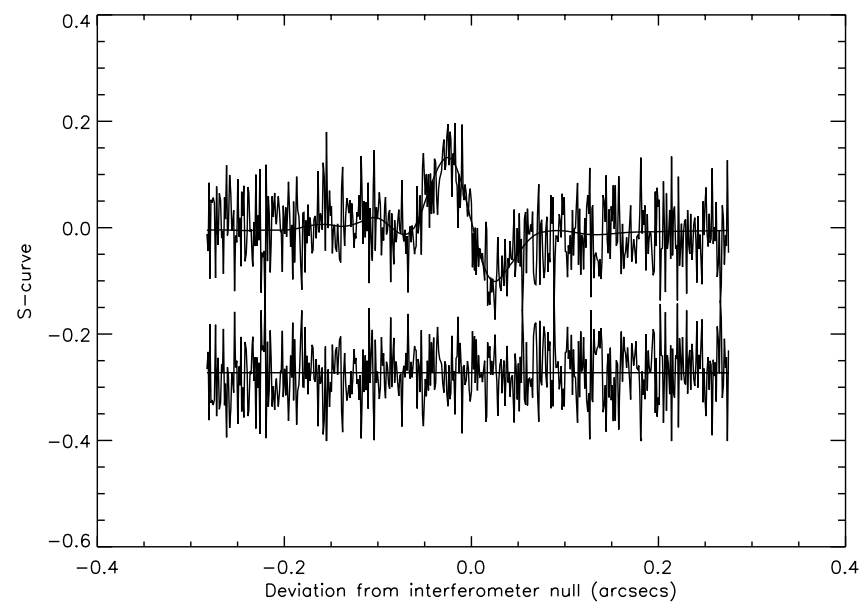

shown in the $\chi^{2}$ values. It seems most likely that this is because of an inadequately matched reference $S$ curve due to variations with time of the S-curve form. This data set is considered further in $\S 6$ above.

\subsection{C 273 (Figure 12)}

These curves also have excellent $\mathrm{S} / \mathrm{Ns}$, and the fits are reasonable, although, like NGC 4151, it appears that better

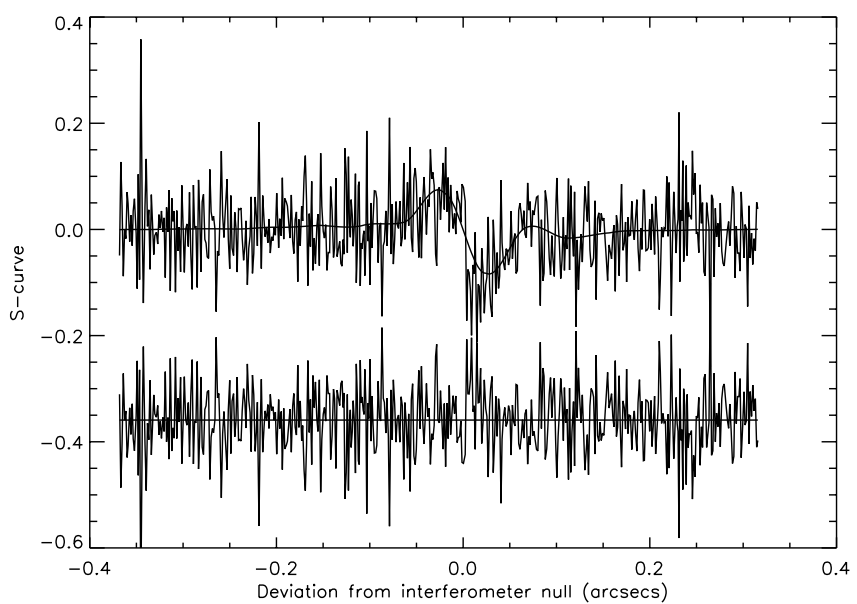

FIG. 8. - Best fits to NGC $1275 x$ and $y$ S curves
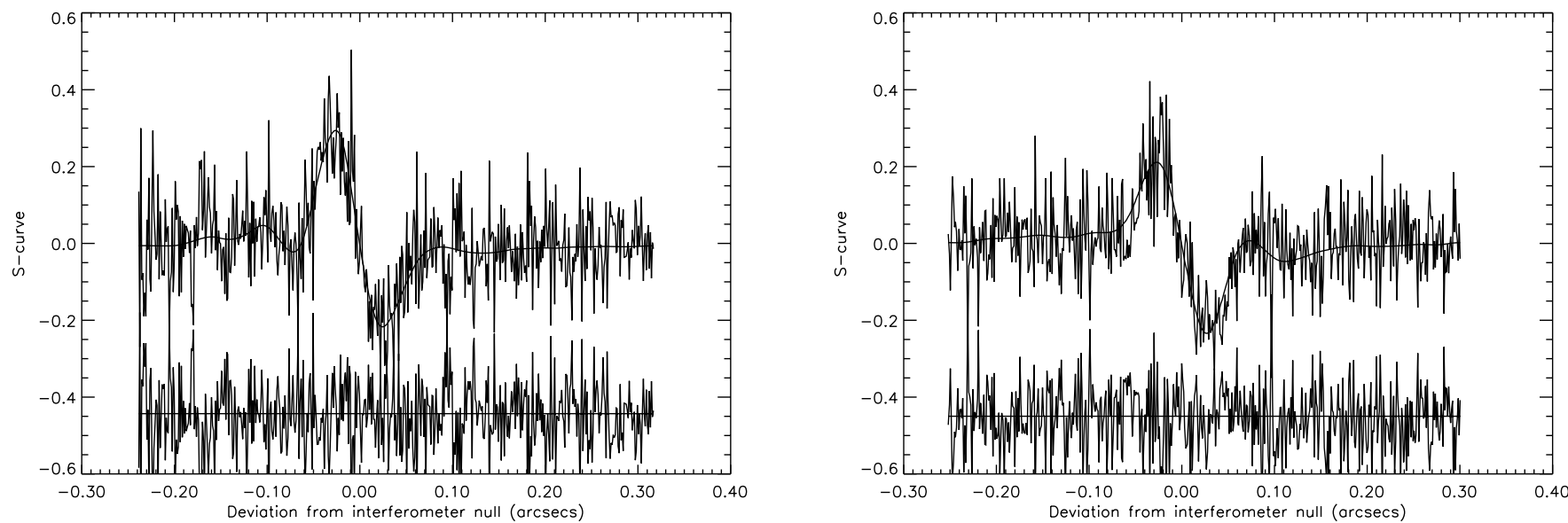

FIG. 9.-Best fit to 3C 3451993 April $12 x$ and $y$ S curves 

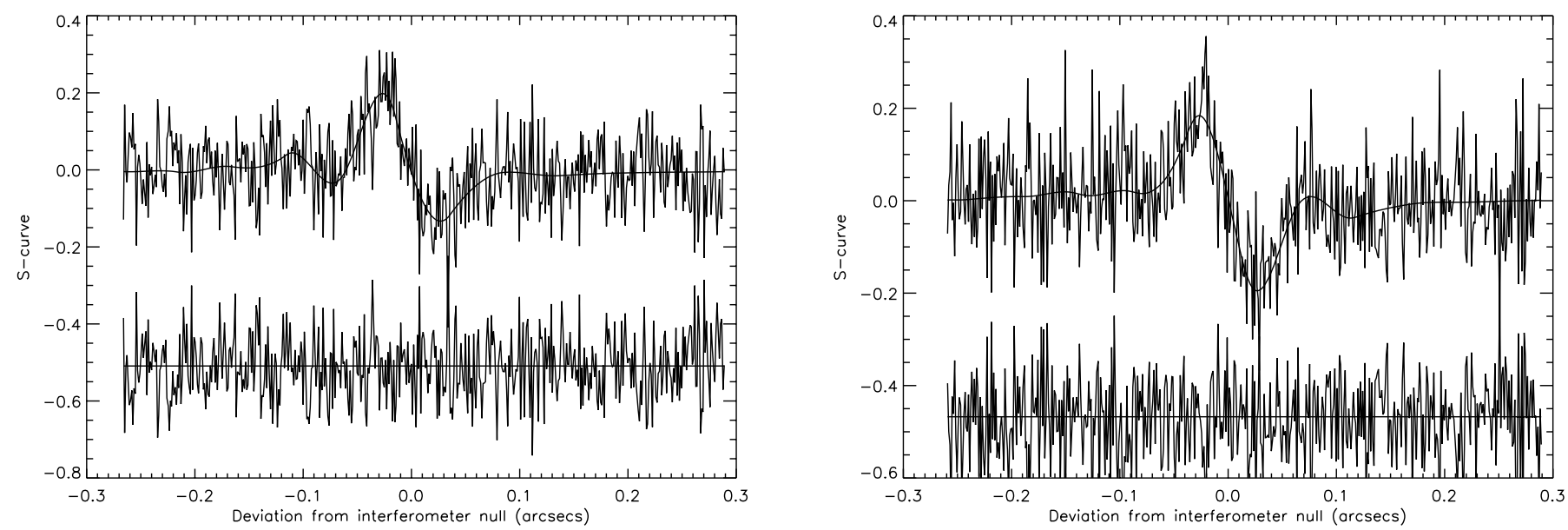

FIG. 10.-Best fit to 3C 3451995 February $22 x$ and $y$ S curves

calibrations would allow more information to be extracted. In terms of amplitude and width these curves are very similar to the calibration, indicating that the relative background is low and that the object is, to first approximation, a point. The $x \mathrm{~S}$ curve, which is less stable than in $y$, shows some systematic differences. Possible explanations for such differences were discussed in $\S 5$ above. The $y \mathrm{~S}$ curve's fit is closer, but there is also a systematic difference.
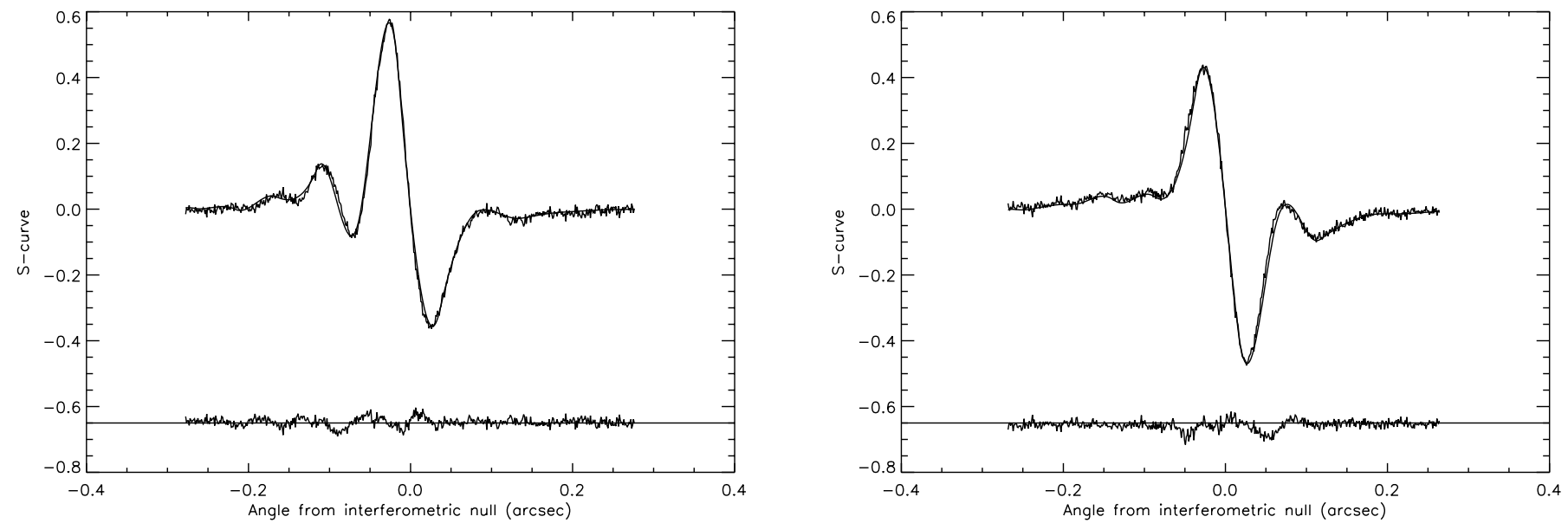

FIG. 11.-Best fit to NGC $4151 x$ and $y$ S curves
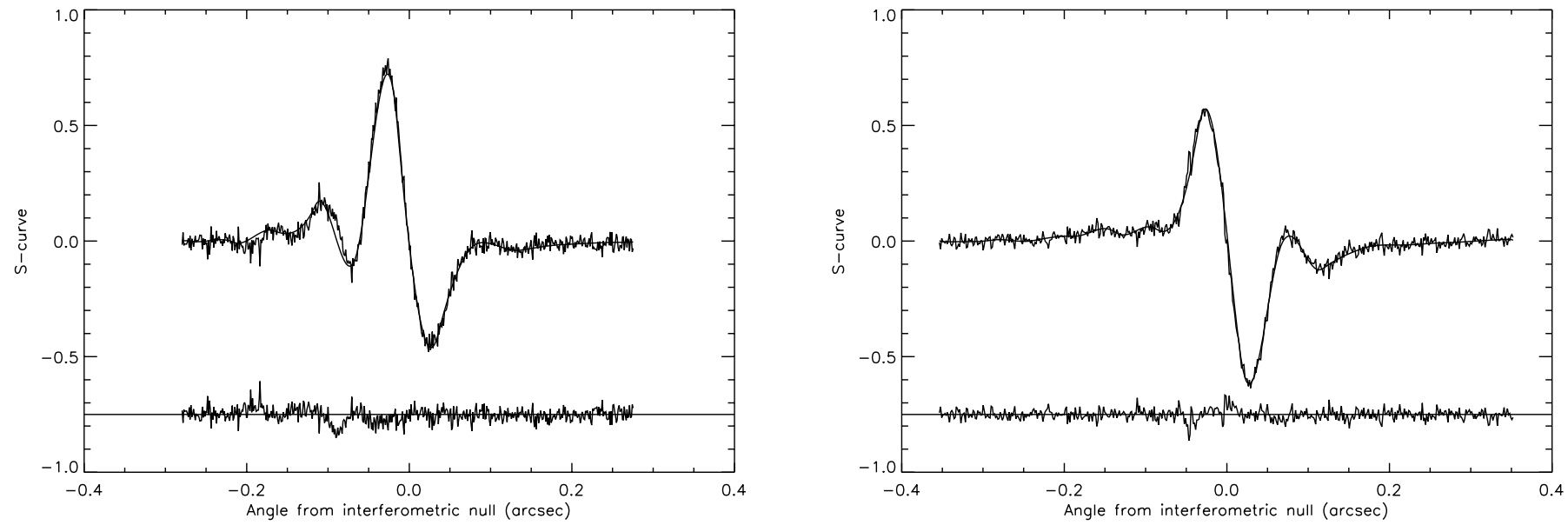

Fig. 12.-Best fit to $3 \mathrm{C} 273 x$ and $y$ S curves 

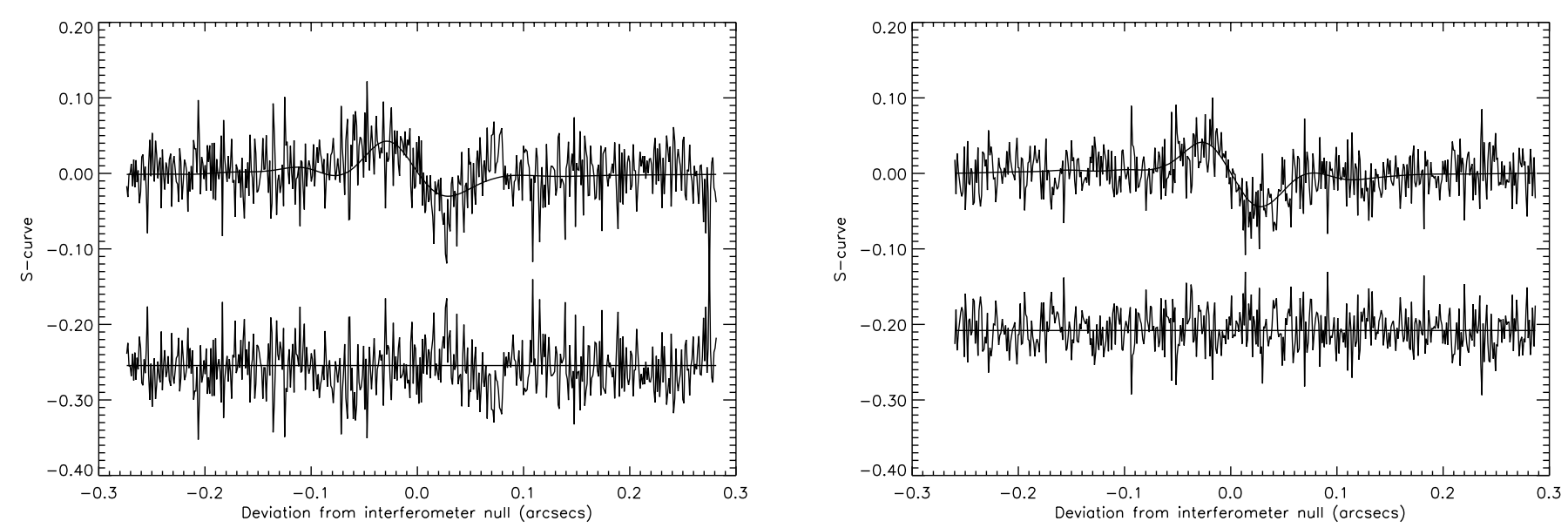

FIG. 13.-Best fit to M87 $x$ and $y \mathrm{~S}$ curves

\subsection{M87 (Figure 13)}

These $S$ curves have very small amplitudes because of the strong background from the underlying elliptical galaxy. The fits are consistent with a point source, but the signal-tonoise ratio is very low.

\section{DISCUSSION}

All the bright AGNs that have been studied using the HST FGS in TRANS mode can be modeled well by assuming that the true intensity distribution of the object is a point source on a background. Statistical tests show that the very small measured widths are not significant. The levels of background measured are consistent with what is expected: bright pointlike objects (e.g., 3C 273) have negligible background and objects that are the cores of bright, large galaxies (e.g., M87 and NGC 4151) have strong backgrounds. For faint objects the instrumental background is detected and appears at the expected strength (e.g., 3C 279).

An assessment of the systematic effects limiting the resolution of the FGS3 interferometer imply that we would not expect to detect extents (FWHM) less than 20 mas $(\sigma=9$ mas). These effects are dominated by the instability of the FGS3 interferometer in combination with the spherically aberrated telescope optics and the difficulty of accurately co-adding individual scans of faint objects.

We may use our results to estimate the physical extents of the emitting regions and the luminosity densities that these imply. Table 5 tabulates these quantities for those objects with statistically significant upper limits. We assume a spherical, uniform, optically thin emitting region, $H_{0}=65$ $\mathrm{km} \mathrm{s}^{-1} \mathrm{Mpc}^{-1}$, and that the upper limit to the radius of the sphere is the measured $\sigma$. The luminosities are calculated from the $V$ magnitude of the objects and the sun with no consideration of color effects. These objects are variable and hence approximate mean values are used for the $V$ magnitude. For the two bright objects we use the limit of $\sigma=9$ mas as deduced from a study of the systematics. For the fainter 3C 279 the noise of the data itself becomes more important, and we use the limit $\sigma=12$ mas. For the brightest objects in our sample the FGS3 observations are limited by the systematic effects described in detail in $\S 5$ above.

Because it is both the closest object in our study and has the $S$ curves with the highest signal-to-noise ratios, the NGC 4151 data yield the smallest physical limit on the size of the nuclear emission region. This object is the closest and most studied Seyfert I galaxy and has been modeled in detail in the framework of the unified view of AGNs by Cassidy \& Raine (1997). The broad-line region (BLR) of this object varies dramatically on timescales of weeks and must have an angular extent below 1 mas. The narrow-line region (NLR) farther out extends over hundreds of parsecs and has been studied in detail from the ground and by imaging with HST. Our FGS observations are not sensitive to these extended features on arcsecond scales; they are included in the measured background. We expect any structure we see on scales of 10 mas to come from material around the BLR that is scattering the intense nuclear radiation. If such scattering were bright enough to effectively broaden the nuclear intensity profile in the optical, we would detect such extent. Because our observations are consistent with a point source, it appears likely that such scattering is overwhelmed by the direct view of the BLR or that such a broadening occurs only closer to the BLR.

\section{CONCLUSIONS}

The original HST FGS3 interferometer has been shown to be capable of measuring extents down to about $\sigma=9$

TABLE 5

Limits for Physical EXtent and Luminosity Density of Emitting Regions Measured with FGS3

\begin{tabular}{|c|c|c|c|c|c|}
\hline Object Name & $z$ & Distance Modulus & Assumed $V$ & Upper Limit Radius of Emitting Region & $\begin{array}{l}\text { Lower Limit of Luminosity Density } \\
\qquad\left(L_{\odot} \mathrm{pc}^{-3}\right)\end{array}$ \\
\hline $3 \mathrm{C} 279$. & 0.536 & 42.0 & 15 & $12 \mathrm{mas}=73 \mathrm{pc}$ & $6.9 \times 10^{6}$ \\
\hline $3 C 273 \ldots \ldots \ldots$ & 0.158 & 39.3 & 12.8 & $9 \mathrm{mas}=26 \mathrm{pc}$ & $4.7 \times 10^{7}$ \\
\hline
\end{tabular}

NoTE.-The performance of the prerefurbishment FGS is limited by systematics on the brighter objects. 
TABLE 6

Estimated Limits for Physical Extent and Luminosity Density of Emitting Regions with POSTREFURBISHMENT FGS1R

\begin{tabular}{ccc}
\hline \hline Object Name & Upper Limit Radius of Emitting Region & $\begin{array}{c}\text { Lower Limit of Luminosity Density } \\
\left(L_{\odot} \mathrm{pc}^{-3}\right)\end{array}$ \\
\hline 3C $279 \ldots \ldots \ldots$. & $12 \mathrm{mas}=73 \mathrm{pc}$ & $6.9 \times 10^{6}$ \\
NGC $4151 \ldots \ldots$. & $5 \mathrm{mas}=0.38 \mathrm{pc}$ & $5.3 \times 10^{9}$ \\
$3 \mathrm{C} 273 \ldots \ldots \ldots$ & $7 \mathrm{mas}=20 \mathrm{pc}$ & $1.0 \times 10^{8}$ \\
\hline
\end{tabular}

NoTE.-Limits that should be attainable with the FGS1R assuming that the performance will be limited primarily by photon statistics rather than systematics of the instrument.

mas, on the order of $1 \mathrm{pc}$ in nearer AGNs. This surpasses the capabilities of the HST Planetary Camera (PC) by close to 1 order of magnitude. The upper limit is due to a combination of photon statistics and systematic effects not predictable in the prerefurbishment FGS. If these objects were reobserved using the postrefurbishment FGS1R, these systematics would be dramatically reduced and the attainable performance would become limited by the photon statistics of the data. This would lower the upper limits that could be placed on the extent of the AGN emitting regions to the values given in Table 6. The refurbished FGS1R, with S curves that are more stable and closer to the theoretically perfect form and that do not require the pupil stop, which degrades both throughput and resolution, will allow detec- tion of structure on scales of 5 mas or smaller on the brighter AGNs.

We are grateful to many people who contributed to this project over many years. Doris Daou and Nicola Caon gave invaluable help with the data reduction. Mario Lattanzi, Pierre Bely, and Sherie Holfeltz provided much useful advice and encouragement in many discussions. We especially thank Ed Nelan for his simulations, his advice about the FGS systematics, and his careful reading of the manuscript.

We would like to acknowledge support from STScI grants GO-02443.01-87A and GO-06578.01-95A. 485,328 